\title{
Evaluating Organizational Configurations
}

\author{
Loris Penserini, Frank Dignum, Virginia Dignum, \\ Huib Aldewereld \\ Institute of Information and Computing Sciences \\ Universiteit Utrecht, The Netherlands \\ Email: \{loris,dignum,virginia,huib\}@cs.uu.nl
}

\author{
Davide Grossi \\ ILLC \\ University of Amsterdam \\ Amsterdam, The Netherlands \\ Email:d.grossi@uva.nl
}

\begin{abstract}
A Multi-Agent System is often conceived as an organization of autonomous software agents that participate into social and evolving structures (e.g., organizational configurations) suitable to deal with highly dynamic environments. Nevertheless, systems based on agent technologies rarely capitalize on their potentials since their systemic properties-e.g., flexibility, robustness and efficiency-are typically only the byproduct of the (AI) techniques deployed at the implementation level, and are neither explicit object of study nor are taken into consideration at a requirements engineering phase. The paper presents a method, based on graph theory, to exactly compare and evaluate software design system configurations in the engineering of multiagent systems. The theoretical results are presented and validated on a crisis management scenario.
\end{abstract}

\section{Introduction}

The increasing use of Multi-Agent Systems (MASs) technologies -in software development process of complex systems - has given rise to social and organizational conceptual abstractions to cope with the engineering of new system requirements. That is, as a MAS is often conceived as an organization of autonomous software agents, the above conceptual abstractions make easier to study and model real organizations in terms of agent societies. However, actual systems based on these technologies rarely capitalize on their potentials since their systemic properties-e.g., flexibility, robustness and efficiency-are typically only the byproduct of the (AI) techniques deployed at the implementation level, and are neither explicit object of study nor are taken into consideration at a requirements engineering phase. This may lead to misalignments between the run-time behavior and users' (requirements) expectations.

To rectify such shortcomings, several agent oriented methodologies have been proposed to support the development of systems that require to be robust, flexible and efficient and to dynamically deal with requirement changes [1]-[3], [7]. An important challenge is to provide a practical and flexible way (e.g., by mathematical tools) to actually discriminate among available system configurations both at design-time - e.g., for requirements analysis purposesand at run-time - e.g., to improve the software agent's decision making process.

In this paper, we propose a general method to correlate the organization's adherence to the general qualities/properties of robustness, flexibility and efficiency with its ability to select the most appropriate (structural) configuration for the context change at hands. Organizations will be studied from a graph theoretical point of view and the three properties of robustness, flexibility and efficiency will be defined in graph theoretical terms in order to allow for an exact characterization of the problem of their maximization.

To deal with the organizational modelling, we adopt the OperA methodology [3] that provides the required social abstractions.

The paper is structured as follows. Section 2 gives an overview of the organizational model for the crisis management scenario by the use of the OperA methodology. Section 3 briefly recalls the organizational measures adopted and extended within our evaluation framework. Section 4 formalizes the concept of organization configuration used to generate some example scenarios. Section 5 illustrates the theoretical framework along with preliminary results generated by an implementation of the proposed evaluation framework. In Section 6, we present some related work. Finally, Section 7 gives some conclusions and points out main future work directions.

\section{Organizational Model for Crisis Manage- ment}

The modelled scenario is inspired by the real case of the Dutch crisis management procedures. These procedures substantially differ depending on the severity level of the incident and are standardized by the Dutch Ministry of Internal Affairs in order to better handle incidents of different scales, from common (traffic) accidents to full-scale (natural) disasters. These levels of severity are five, named GRIP and (briefly) defined as follows. GRIP-0 for routine accidents where no coordination is needed. GRIP-1 related to incidents where multi-disciplinary coordination at the 
operational level is required. GRIP-2 regards large scale incidents that require advanced multi-disciplinary coordination, i.e., a Regional Operational Team and a Municipality Team are created. GRIP-3 deals with disasters involving several regions; hence, a separate regional strategic coordination is established. GRIP-4 regards large scale disasters that imply coordination policies at provincial or national level.

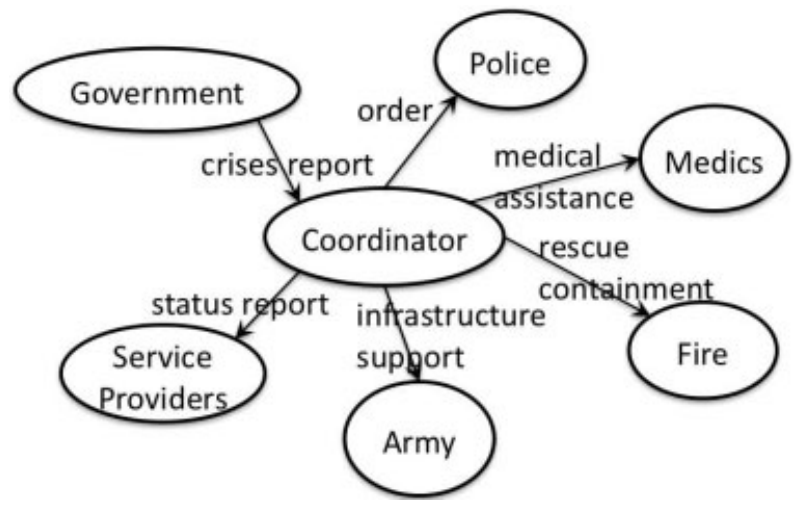

Figure 1. Possible organizational model by OperA's Social Structure diagram.

The domain analysis of the crisis organization is fulfilled at a conceptual level by the use of the OperA agent oriented software engineering methodology [3]. Here, we only focus on the level of OperA Organizational Model by the use of Social Structure diagrams, e.g., as illustrated in Figure 1. The organizational and social structure of Figure 1 represents a (simplified) crisis situation when incident severity is going to scale from GRIP-2 to GRIP-3. The role Coordinator has the power over the other roles in order to coordinate the most convenient intervention to cope with the current evolving incident. For example, Coordinator can delegate the objective medical assistance to the role Medics, as the incident may involve wounded.

Different organizational models, e.g., as the one illustrated in Figure 1, do give a lot of freedom at the operational level to select the best behavior for the specific situation, e.g., agent communication protocols, monitoring and decision-making capabilities. Nevertheless, organizational models present this problem: who does guarantee that the organization of agents society maximizes the organizational properties of robustness, flexibility and efficiency in different scenarios? These properties are strongly connected with the domain knowledge, hence, hard to be grasped by any AI agent at the operational level. In other words, the model should provide the designer with the right balancing of contextual and social information needed for suggesting the best possible configuration the agent society can assume in order to cope with different situations at hands.

The above considerations suggest the key research question of the present paper: given a specific context situation, what is the best (set of) organization(s) to cope with it?

\section{Quantitative Analysis of Organizational Structures}

In this Section, we provide an overview of the idea proposed in [4], [5] that consists in modelling the organizational structures along with their properties -i.e., robustness, flexibility and efficiency — via directed graphs, which naturally fit our paper's aim.

Organizational structures. An important modelling aspect is that each organization mainly concerns three structural dimensions of power, coordination and control. The power structure defines the task/objective delegation patterns possible within the organization. The coordination structure concerns the flow of knowledge within the organization. The control structure deals with task recovery functions of the organization. Given the above considerations an organizational structure may be defined as follows.

Definition 1. An organizational structure OS is a tuple:

$$
\left\langle\text { Roles }, R_{\text {pow }}, R_{\text {coord }}, R_{\text {contr }}\right\rangle
$$

where Roles is a finite set of roles, and $R_{\text {pow }}, R_{\text {coord }}, R_{\text {contr }}$ are three irreflexive binary relations on Roles characterizing, respectively, the Power, the Coordination and the Control structures. In addition, we impose the following constraints ${ }^{1}$ :

$i)(r, s) \in R_{\text {Pow }} \Rightarrow$ there exists a $R_{\text {Coord }}-$ path from $r$ to $s$; $i i)(r, s) \in R_{\text {Pow }} \Rightarrow$ there exists a $t \in$ Roless.t. $R_{\text {Contr }}(t, s)$.

For every $R_{k}$ s.t. $k \in\{$ pow, coord, contr $\}$, we denote with Roles $_{k}$ the smallest subset of Roles such that, if $(x, y) \in R_{k}$ then $x, y \in$ Roles $_{k}$. That is, each Role Renotes the set involved in the structural dimension $k$; hence, each digraph $\left\langle\right.$ Roles $\left._{k}, R_{k}\right\rangle$ represents the structural dimension $k$ for the organization. As to the constraints, they simply state that the occurrence of a power relation between role $r$ and role $s$ requires: i) the existence of a (finite) coordination path from $r$ to $s$ so that effective informative actions can transmit the relevant knowledge of agents enacting role $r$ to agents enacting role $s$; and ii) the existence of at least an element $t$ (which, notice, might be $r$ itself) which is in a control relation with $s$.

Basic notions from graph theory. An $R_{k}$-path (of length $n)$ is a sequence $\left\langle x_{1}, \ldots, x_{n}\right\rangle$ of distinct elements s.t. $\forall x_{i} \in$ Roles, $1 \leq i<n,\left(x_{i}, x_{i+1}\right) \in R_{k}$. An $R_{k}$-semipath (of length $n$ ) is a sequence $\left\langle x_{1}, \ldots, x_{n}\right\rangle$ of distinct elements s.t. $\forall x_{i} \in$ Roles, $1 \leq i<n,\left(x_{i}, x_{i+1}\right) \in R_{k}$ or $\left(x_{i+1}, x_{i}\right) \in$ $R_{k}$. Moreover, the indegree $i d_{k}(d)$ of a role $d$ in structure $k$ (i.e., $d \in$ Roles $\left._{k}\right)$ is the number of roles $d_{1}$ s.t. $\left(d_{1}, d\right) \in R_{k}$; similarly follows that the outdegree $\operatorname{od}_{k}(d)$ of a role $d$ in structure $k$ (i.e., $d \in$ Roles $_{k}$ ) is the number of roles $d_{1}$ s.t. $\left(d, d_{1}\right) \in R_{k}$. Our approach fits well with the following organization's structure definition.

1. In [5], organizations satisfying these constraints are called sound. In the present paper we thus consider only sound organizations. 


\begin{tabular}{|c|c|c|}
\hline 1 & Completeness $_{k}(O S)=\frac{\left|R_{k}\right|}{\mid \text { Roles }_{k} \mid \cdot\left(\mid \text { Roles }_{k} \mid-1\right)}$ & Connectedness $_{k}(O S)=1-\frac{\left|D^{\prime S C O N_{k} \mid}\right|}{\mid \text { Roles }_{k} \mid \cdot\left(\mid \text { Roles }_{k} \mid-1\right)}$ \\
\hline 2 & Economy $_{k}(O S)=1-\frac{\left|R_{k}\right|-\left(\mid \text { Roles }_{k} \mid-1\right)}{\mid \text { Roles }_{k} \mid \cdot\left(\mid \text { Roles }_{k} \mid-1\right)-\left(\mid \text { Roles }_{k} \mid-1\right)}$ & \\
\hline 3 & Unilaterality $_{k}(O S)=1-\frac{\left|S I M_{k}\right|}{\left|R_{k}\right|}$ & Univocity $_{k}(O S)=\frac{\mid \text { IN }_{k} \mid}{\mid \text { Roles }_{k} \mid}$ \\
\hline 4 & InCover $_{j, k}(O S)=\frac{\left|I N_{j}^{+} \cap I N_{k}^{+}\right|}{\left|I N_{k}^{+}\right|}$ & OutCover $_{j, k}(O S)=\frac{\left|O U T_{j}^{+} \cap O U T_{k}^{+}\right|}{\left|O U T_{k}^{+}\right|}$ \\
\hline 5 & $\operatorname{Chain}_{j, k}(O S)=\frac{\left|I N_{j}^{+} \cap O U T_{k}^{+}\right|}{\left|O U T_{k}^{+}\right|}$ & $\operatorname{Overlap}_{j, k}(O S)=\frac{\left|L I N K_{j, k}\right|}{\left|R_{k}\right|}$ \\
\hline
\end{tabular}

Table 1. Measures required to characterize the properties of Robustness, Flexibility and Efficiency.

In the following, we briefly recall the principal measures defined in [5] and summarized in Table 1, based on graph theory, required to build the organizational properties.

Completeness, Connectedness and Economy. In practice, by means of completeness and connectedness, we are interested in determining how strongly roles are linked with one another within one of the structural dimensions $k$. Worth noticing is that by the connectedness we can discover cutpoints, namely, roles whose removal may dramatically decreases the value of this parameter. The first row in Table 1 shows the formulae for Completeness and Connectedness where $\left|R_{k}\right|>0$ and DISCON $N_{k}$ is the set of ordered pairs of $(x, y) \in$ Roles $_{k}$ s.t. there is neither a $R_{k}$-semipath from $x$ to $y$ nor from $y$ to $x$; while, $\left|R_{k}\right|>0$ states that the structural dimension $k$ does indeed exist. The second row in Table 1 shows the formula for Economy that measures a tradeoff between connectedness and completeness. The intuition behind the Economy is that the most economical structure is a connected structure which minimizes the number of links. The optimal value for the Economy $(O S)$ is given when $\left|R_{k}\right|=n-1, \mid$ Roles $_{k} \mid=n$ (only one link for each role), that is, Economy $(O S)=1$.

Unilaterality and Univocity. These two measures are relevant, on the one hand, to observe the level of subordination in a structure by looking at the orientation of its links (unilaterality) and, on the other hand, to determine the level of conflicts and redundancies in a given structure (univocity). For example, in $R_{\text {coord }}$ dimension, the higher is the value of unilaterality, the lower is the amount of 'peer-topeer' based information exchange within OS. The formulae for Unilaterality and Univocity have been illustrated in the third row of Table 1 where $\left|R_{k}\right|>0$ and $S I M_{k}$ is the set of symmetric pairs $(x, y) \in R_{k}$ s.t. $(y, x) \in R_{k}$; hence, $\left|S I M_{k}\right|$ is twice the number of symmetric pairs. $I N_{k}$ denotes the set of roles $(x)$ in the dimension $k$ s.t. $i d_{k}(x)=0$ or $i d_{k}(x)=1$ (say for brevity $i d_{k}(x) \leq 1$ ).

Cover, Chain and Overlap. This group of measures deals with correlations between different structural dimensions. The InCover and OutCover describe respectively how many incoming and outgoing links for each role in $k$ are also present for the same role in $j$. In particular, the formulae for InCover and OutCover have been illustrated in the fourth row of Table 1 where $\left|R_{k}\right|>0$ and $I N_{i}^{+}$is the set of roles $x \in$ Roles $_{i}$ s.t. $1 \leq i d_{i}(x)$; while, $O U T_{i}^{+}$is the set of roles $x \in$ Roles $_{i}$ s.t. $1 \leq \operatorname{od}_{i}(x)$. While, the formulae for Chain and Overlap have been showed in the fifth row of Table 1 where $\left|R_{k}\right|>0$ and $I N_{i}^{+}$and $O U T_{i}^{+}$as above; while, $L I N K_{j, k}$ is the set of pairs $(x, y)$ s.t. $(x, y) \in R_{j}$ and $(x, y) \in R_{k}$, i.e., $L I N K_{j, k} \equiv R_{j} \cap R_{k}$. The Chain concerns the number of roles that, on the one hand, are recipient of, e.g., obligations/tasks (incoming links) within a dimension $j$ and, on the other hand, are addressing other roles with, e.g., information (outgoing links), within another dimension $k$. As the set $L I N K_{j k}$ deals with the numbers of pairs that are in common between $j$ and $k$, the Overlap $_{j k}$ gives the degree of overlap of the two structures.

One of the main contributions of the work presented in [5] has been to interpret and to adopt the above measures within an organizational setting - to evaluate an organization with respect to its level of adherence to the properties of robustness, flexibility and efficiency. Here we briefly recall the main intuitions behind the characterization of these three organizational properties.

Robustness. As pointed out in [5], robustness asks for redundancy in the power and coordination structural dimensions needed for distributing tasks within an organization. For example, within the framework of structural properties, this requirement can be translated to a low degree of Univocity $_{\text {pow }}$ and Unilateralitycoord. This latter allows to increase the bilateral negotiations of tasks by allowing for symmetric links, and thereby replacing direct delegations; intuitively, this also leads to have a high value for Overlap coord,pow.

Flexibility. In real organizations, as well as in agent societies, roles' capabilities are diversified, therefore flexibility is related to the ability of an organizational structure to deal with changing tasks [5]. Intuitively, to deliver on this latter aim, the power structure should not be too 


\begin{tabular}{|c|c|c|c|c|c|}
\hline \multicolumn{2}{|l|}{ Robustness } & \multicolumn{2}{|l|}{ Flexibility } & \multicolumn{2}{|l|}{ Efficiency } \\
\hline Overlap Coord-Pow & 1 & Completeness Pow & 0 & Connectedness Pow & 1 \\
\hline Chain $_{\text {Contr-Pow }}$ & 1 & Connectedness ${ }_{\text {Pow }}$ & 0 & Economy & 1 \\
\hline Chain Contr-Coord $_{\text {Con }}$ & 1 & Chain $_{\text {Contr-Pow }}$ & 1 & Economy $y_{\text {Coord }}$ & 1 \\
\hline InCover Contr-Coord & 1 & Completeness $_{\text {Coord }}$ & 1 & Overlap Coord-Pow & 1 \\
\hline OutCover Pow-Contr & 1 & Connectedness Coord & 1 & Overlap $P$ ow-Coord & 1 \\
\hline OutCover Pow-Coord & 1 & OutCover Pow-Contr & 1 & Unilaterality ${ }_{\text {Pow }}$ & 1 \\
\hline Completeness $s_{\text {Coord }}$ & 1 & & & UnivocityPow & 1 \\
\hline Connectedness $s_{\text {Coord }}$ & 1 & & & Economy Contr & 1 \\
\hline UnivocityPow $_{\text {P }}$ & 0 & & & Overlap Contr-Pow & 1 \\
\hline Unilaterality $_{\text {Coord }}$ & 0 & & & Overlap Pow-Contr & 1 \\
\hline Univocity $_{\text {Contr }}$ & 0 & & & & \\
\hline Flatness Contr & 0 & & & & \\
\hline
\end{tabular}

Table 2. Measures and their optimal values for the maximization of robustness, flexibility and efficiency.

articulated, forcing to distribute tasks towards predefined patterns. Hence, e.g., low degrees of Completeness $s_{\text {pow }}$ and Connectedness pow suggest themselves. As already said for the robustness, also for the flexibility a fault tolerance ability should hold.

Efficiency. There is general agreement that the higher is the number of links between roles in an organization, the less efficient is its performance, since link does not come without costs [5]. However, a trade-off is obviously desirable. For instance, a paradigmatically efficient power structure is the tree structure.

The above considerations have been summarized in Table 2 . The table provides the three sets of properties along with the values whose maximization also positively contributes to the maximization of the corresponding set (for details see [5]).

Worth noticing that the three properties cannot be maximized all together by simply handling their internal measures. For example, the action of decreasing Univocity Uow $_{\text {ow }}$ in order to maximize Robustness causes the opposite effect towards the property Efficiency. The same effect holds between Flexibility and Efficiency trying to maximize the measure Connectedness Pow.

\section{Choosing an Organizational Configuration}

Here we are interested in characterizing, at a structural level, those organizational configurations that are compliant with the selected model, and which better fit the crisis situation at hands. To deliver on the aim of this Section, we introduce the concept of organization's configurations simply adapting to the previous definition 1 of organizational structure.

Definition 2 (Organizational configurations). Each organizational model $(j)$ defines a finite set of organizational configurations $o c_{i}^{j}$ as follows:

$$
o c_{i}^{j}=\left\langle\text { Roles }^{i}, R_{\text {pow }}^{i}, R_{\text {coord }}^{i}, R_{\text {contr }}^{i}\right\rangle^{j}
$$

where each $o c_{i}^{j}$ is an organizational structure (see Definition 1), $1 \leq j \leq m$ represents the selected organizational model and $1 \leq i \leq n$ identifies each single configuration within the model $j$.

Despite the proposed evaluation framework can be adopted to discriminate among models, for the sake of simplicity, in this paper we illustrate the approach focusing on the organizational model of Figure 1 that allows for a set of configurations that differently impact on possible scenarios/situations. Figure 2 and 3 provide two possible sets of configurations to respectively deal with two different scenarios, as detailed below.

Scenario 1. While the crisis organization is involved in a GRIP-2 level of a floodwater incident, Coordinator comes to know about blocked roads that hamper the incident access, because the heavy rainy causes the increasing of floodwater and the threatening of the whole region. This context change also forces the Government to scale up to the GRIP-3 level.

The organizational model — partially illustrated by Figure 1- is abstract enough to support (at structural level) the scaling up from GRIP-2 to GRIP-3. In fact, the major changes within the organization specification regard a new set of competencies assigned to the role Coordinator that gets the executive authority to decide when activate the role Army intervention along with the required resources (e.g., from simple manpower to complex infrastructures). Configurations $o c_{2}$ and $o c_{3}$ are more suitable for situations that occur in Scenario 1 when the emergency level GRIP3 is permanent. The main differences between these latter are that $o c_{3}$ allows for coordination and control links also between Police and Army.

Scenario 2. While the crisis organization is facing with a floodwater incident at level of GRIP-2, Coordinator receives conflicting reports about the crisis situation, e.g., wrong levels of water and places with wounded to be rescued soon.

The situation illustrated in Scenario 2 may lead Coordinator to perform a wrong risk analysis (e.g., within an incident assessment activity) causing dangerous consequences to the whole organization. Figure 3 shows that despite $o c_{1}$ (i.e., the same adopted within Scenario 1) 

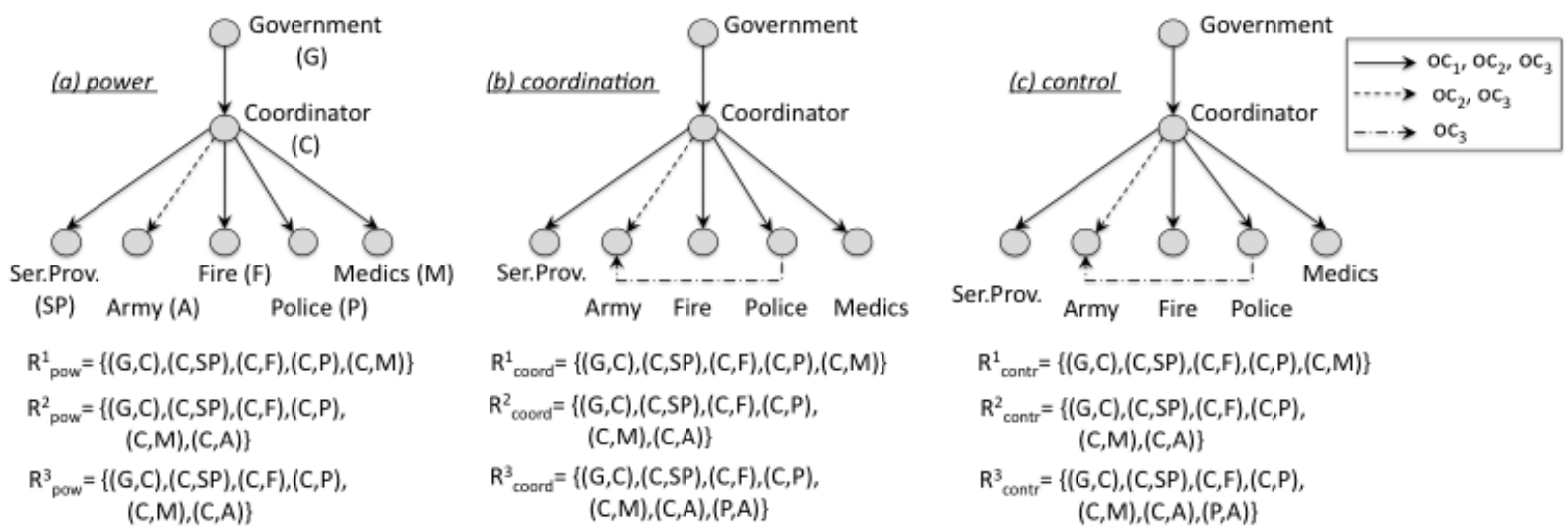

Fiaure 2. Possible oraanizational confiaurations for dealina with Scenario 1.
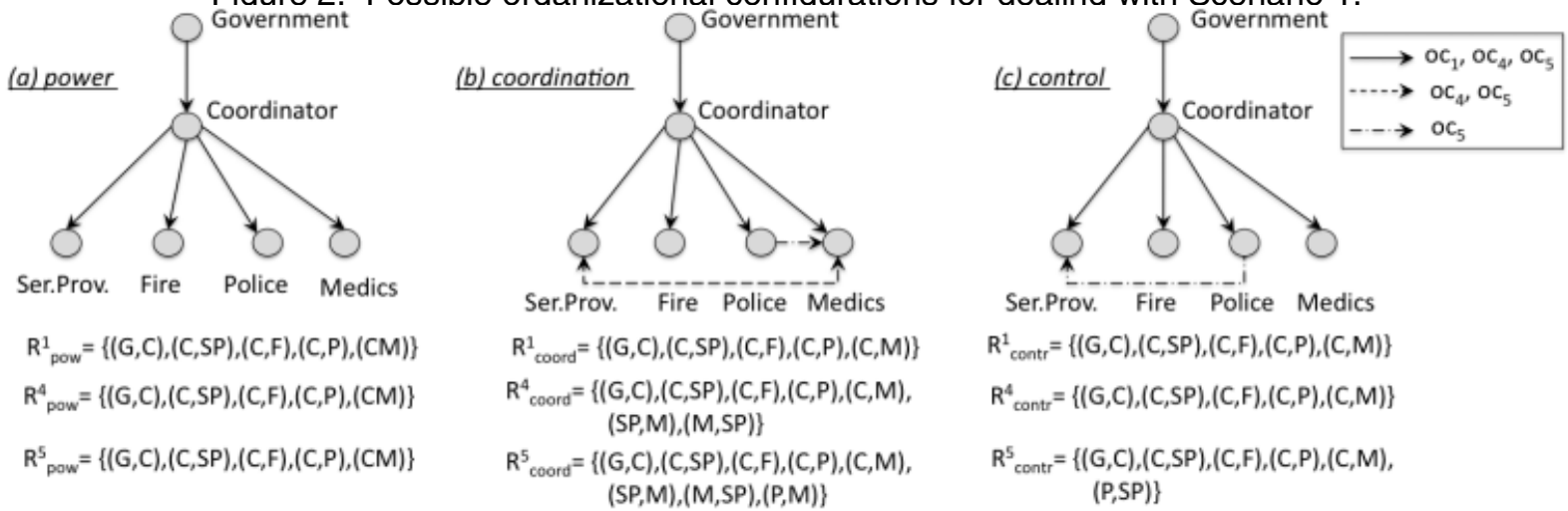

Figure 3. Possible organizational configurations for dealing with Scenario 2 at GRIP-2.

is suitable to deal with the most of situations at GRIP-2, does not perform well to cope with conflicting reports, as needed by Scenario 2. As shown in Figure 3, the configuration $o c_{4}$ introduces - with respect to $o c_{1}-$ the symmetric coordination link between roles Medics and Service Providers, guaranteeing a shared knowledge in terms of information flows between the two roles. This latter may avoid conflicting reports about numbers and locations of wounded to be rescue. Moreover, Scenario 2 may also occur because reports arrive from different stakeholders, such as the weather forcasting unit (waterways management agency in the Netherlands) and citizens describe/perceive different levels of floodwater for the same location. This possible cause of conflicting reports can be contrasted, on the one hand, by enforcing the control level over the Service Provider activities by the Police authority and, on the other hand, allowing Police to coordinate with Medics, as modelled by $o c_{5}$ in Figure 3.

\section{Evaluating Organizational Configurations}

\subsection{Into the structural dimensions}

The evaluation framework we propose here aims at quantifying the quality of a set of organizational configurations.
Considering Definition 2 and the set of structural measures discussed in Section 3, the designer can provide a first evaluation for every organizational configuration and organize them in tables for each property -i.e., Robustness, Flexibility and Efficiency - as partially displayed by Table 3 in the next section.

A first step in our framework is to evaluate the total adherence of each configuration towards each single property, considering its closeness to the ideal value proposed in Table 2 of Section 3.

Let $\mathcal{P}^{x}=\left\{P_{1}^{x}, \ldots, P_{n}^{x}\right\}$ be the set of measures for the property $x \in\{R, F, E\}$ where $R$ stands for Robustness, $F$ for Flexibility and $E$ for Efficiency. Notice that each $\mathcal{P}^{x}$ represents thus a column in Table 2. Now, each of these sets $\mathcal{P}^{x}$ can be bipartitioned in $\left(\mathcal{P}_{0}^{x}, \mathcal{P}_{1}^{x}\right)$ where $\mathcal{P}_{0}^{x}$ is the set of measures whose optimal value for $x$ is 0 and, $\mathcal{P}_{1}^{x}$ is the set of measures whose optimal value is, instead, 1. By means of this bipartition, we can keep track of the respective optimal values of each parameter within a property, i.e., of the $1 \mathrm{~s}$ and 0 s occurring in Table 2. This preparation allows for the following definition.

Definition 3 (Value of a property). Let $o c$ be an organizational configuration as in Definition 2. The value of a property $x \in\{R, F, E\}$ for $o c$ is defined by the following equation: 


\begin{tabular}{|c|c|c|c|c|c|c|}
\hline Efficiency & $o c_{1}$ & $O c_{2}$ & $o c_{3}$ & $O c_{4}$ & $O c_{5}$ & ideal \\
\hline Connectedness Pow & 1 & 1 & 1 & 1 & 1 & 1 \\
\hline Economy $_{\text {Pow }}$ & 1 & 1 & 1 & 1 & 1 & 1 \\
\hline Economy $y_{\text {Coord }}$ & 1 & 1 & 0,9722 & 0,92 & 0,88 & 1 \\
\hline Overlap Coord-Pow & 1 & 1 & 1 & 1 & 1 & 1 \\
\hline Overlap Pow-Coord & 1 & 1 & 0,8571 & 0,7143 & 0,625 & 1 \\
\hline Unilaterality ${ }_{\text {Pow }}$ & 1 & 1 & 1 & 1 & 1 & 1 \\
\hline UnivocityPow & 1 & 1 & 1 & 1 & 1 & 1 \\
\hline Economy $y_{\text {Contr }}$ & 1 & 1 & 0,9722 & 1 & 0,96 & 1 \\
\hline Overlap Contr-Pow & 1 & 1 & 1 & 1 & 1 & 1 \\
\hline Overlap Pow-Contr & 1 & 1 & 0,8571 & 1 & 0,8333 & 1 \\
\hline InBalance $P$ ow & 0,2 & 0,1667 & 0,1667 & 0,2 & 0,2 & 0 \\
\hline OutBalance Pow & 0,6 & 0,6667 & 0,6667 & 0,6 & 0,6 & 0 \\
\hline InBalance Contr & 0,2 & 0,1667 & 0,3333 & 0,2 & 0,4 & 0 \\
\hline OutBalance Contr & 0,6 & 0,6667 & 0,8333 & 0,6 & 0,8 & 0 \\
\hline$E\left(o c_{i}\right)$ by Formula (1) & 0,0552 & 0,0575 & 0,0984 & 0,0867 & 0,1294 & 0 \\
\hline
\end{tabular}

Table 3. Parameter values to characterize the property Efficiency for each configuration $\left(E\left(o c_{i}\right)\right)$.

$$
x(o c)=\alpha \cdot\left(\beta \cdot \sum_{p \in \mathcal{P}_{0}^{x}}\|p(o c)\|+\gamma \cdot \sum_{q \in \mathcal{P}_{1}^{x}}\|1-q(o c)\|\right)
$$

where $\alpha=\frac{\left|\mathcal{P}^{x}\right|}{\left|\mathcal{P}_{1}^{x}\right|^{2}+\left|\mathcal{P}_{0}^{x}\right|^{2}}, \beta=\frac{\left|\mathcal{P}_{0}^{x}\right|}{\left|\mathcal{P}^{x}\right|}$ and $\gamma=\frac{\left|\mathcal{P}_{1}^{x}\right|}{\left|\mathcal{P}^{x}\right|}{ }^{2}$.

Leaving technicalities aside, Formula 1 calculates the values of robustness, flexibility and efficiency of a given $o c$ by taking the sum, normalized by $\alpha$, of the absolute value of all parameters that should tend to 0 plus the sum of the absolute value of the differences from 1 of all parameters that should tend to one, after they have also been normalized by quantities related to the partition dimensions (i.e. $\beta$ and $\gamma$ ). Intuitively, the closer each value of $R(o c), F(o c)$ and $E(o c)$ is to zero, the higher is the adherence of the configuration to each of those properties. The last row of Table 3 gives an example of Formula 1 applied to Efficiency's measures within several configurations $\left(E\left(o c_{i}\right)\right)$.

It is worth spending a few words about the motivation and the (engineering) process that lead us to Formula 1. At the beginning, we considered the simpler version of Formula 1 with $\alpha=\frac{1}{\mathcal{P}^{x} \mid}$ and $\beta=\gamma=1$. Such formula would simply add the two sums and normalize them by the number of measures in the property, i.e., the cardinality of $\mathcal{P}^{x}$. However, this choice does not properly work in specific cases. To appreciate this, consider two configurations $o c_{1}$ and $o c_{2}$ both having two parameters $p$ and $q$ getting the same value and such that they differ only for those parameters, i.e., $p\left(o c_{1}\right)=q\left(o c_{1}\right) \neq p\left(o c_{2}\right)=q\left(o c_{1}\right)$. Now, if $p \in \mathcal{P}_{1}^{x}$ and $q \in \mathcal{P}_{0}^{x}$, then the suggested formula would not be able to compare $o c_{1}$ and $o c_{2}$ since $\left\|1-q\left(o c_{1}\right)\right\|+\left\|p\left(o c_{1}\right)\right\|=$ $\left\|1-q\left(o c_{2}\right)\right\|+\left\|p\left(o c_{2}\right)\right\|=1$. Formula 1 solves this issue by differently weighting the two sums based on the bipartition. As each measure in a property has the same relevance, each sum has to differently contribute towards the total amount

2. We chose this less simplified mathematical style, to make more explicit the intuitions behind it. Despite current partition $\mathcal{P}_{0}^{x}$ contains only positive elements, Formula 1 wants to remain valid also for elements that may range in $[-1,0]$ interval. resulting from this formula. The current choice of $\beta$ and $\gamma$ captures precisely this intuition. ${ }^{3}$ Finally $\alpha$ has been chosen to guarantee the formula to range within the interval $[0,1]$.

Nevertheless, depending on the domain expertise of the designer and/or on costs/risks analysis, some properties may result more relevant than others within specific contexts, independently from the values of the properties' parameters. That is, the intuitive idea is that configurations can be ranked not only according to their (objective) structural properties but also reflecting stakeholders expectations/preferences, as follows:

Definition 4 (Rank of a configuration). Let $o c$ be an organizational configuration as in Definition 2. The rank of $o c$ is calculated according to the following equation:

$\operatorname{rank}(o c)=\rho \cdot\|1-R(o c)\|+\varphi \cdot\|1-F(o c)\|+\eta \cdot\|1-E(o c)\|$

where $\rho, \varphi, \eta \in \mathbb{R}_{\geq 0}$ and $\rho+\varphi+\eta=1$.

Intuitively, $\rho, \varphi$ and $\eta$ are values that weight the importance of the related properties within a specific scenario allowing for a more sensitive context-dependent weighting. Notice that, when the configuration maximizes all the properties according to Formula 1 (i.e., $R(o c)=F(o c)=$ $E(o c)=0)$ and such properties are equally important to deal with the underlying situation (i.e., $\rho=\varphi=\eta=\frac{1}{3}$ ) then $\operatorname{rank}\left(o c_{i}\right)=1$. It is also worth noticing that a lookup table may be provided indicating for each situation the corresponding best set of weights $(\rho, \varphi$ and $\eta)$ to be used to rank the set of configurations in each situation.

Formula 2 provides a straightforward way to compare different configurations on the ground of Formula 1. A finergrained criterion to compare possible configurations on the ground of Formula 1 is Pareto optimality. This concept, defined by V. Pareto over a century ago, is very suitable to cope with problems related to finding a general solution

3. Notice that, in the worst case, the quantity within round brackets in Formula 1 is equal to $\left|\mathcal{P}_{1}^{x}\right|^{2}+\left|\mathcal{P}_{0}^{x}\right|^{2}$. 


\begin{tabular}{|c|c|c|c|c|c|c|c|c|c|c|c|}
\hline \multicolumn{6}{|c|}{ Scenario 1: GRIP-2 $\rightarrow$ GRIP-3 } & \multicolumn{6}{|c|}{ Scenario 2: conflicting in GRIP-2 } \\
\hline conf. & $R\left(o c_{i}\right)$ & $F\left(o c_{i}\right)$ & $E\left(o c_{i}\right)$ & $\operatorname{rank}\left(o c_{i}\right)$ & P.opt. & conf. & $R\left(o c_{i}\right)$ & $F\left(o c_{i}\right)$ & $E\left(o c_{i}\right)$ & $\operatorname{rank}\left(o c_{i}\right)$ & P.opt. \\
\hline$O c_{1}$ & 0,3417 & 0,3833 & 0,0552 & 0,7399 & $\sqrt{ }$ & $o c_{1}$ & 0,3417 & 0,3833 & 0,0552 & 0,7399 & $\sqrt{ }$ \\
\hline$o c_{2}$ & 0,3429 & 0,3857 & 0,0575 & 0,7380 & - & $O c_{4}$ & 0,3457 & 0,3700 & 0,0867 & 0,7325 & $\sqrt{ }$ \\
\hline$o c_{3}$ & 0,3905 & 0,4476 & 0,0984 & 0,6878 & - & $O c_{5}$ & 0,3825 & 0,4300 & 0,1294 & 0,6860 & - \\
\hline
\end{tabular}

Table 4. Ranking of configurations according to the evaluation criteria having chosen all weights equal to $\frac{1}{3}$.

to multiple objectives optimization, where the approaches devoted to seek for a global objective function do not perform well [1], [8].

Definition 5 (Dominant configurations). Let $O C=$ $\left\{o c_{1}, \ldots, o c_{n}\right\}$ be a set of possible organizational configurations, let $x \in\{R, F, E\}$ and let $o c_{i}, o c_{j} \in O C$ be any two distinct configurations. We say that $o c_{i}$ dominates $o c_{j}$ if $\forall x \in\{R, F, E\}, x\left(o c_{i}\right) \geq x\left(o c_{j}\right)$ and $\exists x \in\{R, F, E\}$ such that $x\left(o c_{i}\right)>x\left(o c_{j}\right)$.

Definition 6 (Pareto optimal configurations). Let $O C=$ $\left\{o c_{1}, \ldots, o c_{n}\right\}$ be a set of possible organizational configurations. A configuration $o c_{i} \in O C$ is said to be Pareto optimal if there does not exist an $o c_{j} \in O C$ such that $o c_{j}$ dominates $o c_{i}$.

In practice, Pareto optimality complements the ranking criteria given by Formula 2 by allowing for further filtering the configurations with highest rank.

\subsection{Evaluating the crisis management organization}

The proposed evaluation framework for organizational configurations has been implemented and used to produce a preliminary set of experiments. This section reports on some of the results obtained by applying our framework to the crisis management scenario described in Section 4.

Such application gave us an useful feedback for validating and fine-tuning the theoretical framework itself. For example, within specific organizational settings (star-like configurations), we observed that little changes within the structures of power and control -which cause an increase in the number of incoming and/or outcoming dependencies for a single node (role) - were not sensed by the parameters of Efficiency. Here, we only report on the two new parameters/measures that have been added within the property Efficiency to cope with such changes in the power and control structures.

$$
\begin{aligned}
& \text { InBalance }_{k}(\text { oc })=\frac{\operatorname{maxID}_{k}-\operatorname{minI} D_{k}}{\mid \text { Roles }_{k}-1 \mid} \\
& \text { OutBalance }_{k}(\text { oc })=\frac{\text { maxOD }_{k}-\min O D_{k}}{\mid \text { Roles }_{k}-1 \mid}
\end{aligned}
$$

where $1<\mid$ Roles $_{k} \mid$ and $\operatorname{maxI}_{k}=\max \left\{i d_{k}(d) \mid d \in\right.$ Roles $\left._{k}\right\}$ and $\operatorname{minI}_{k}=\min \left\{i d_{k}(d) \mid d \in\right.$ Roles $\left._{k}\right\}$ and

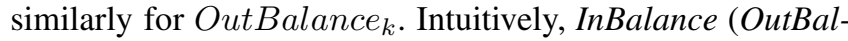
ance) measures how unevenly the work-load -interpreted as the amount of incoming (or outgoing) links- is distributed among nodes of the given structure. When $\max I D_{k}=$

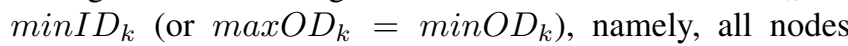
have precisely one incoming (respectively, outgoing) $k$-link, then the InBalance $_{k}$ (respectively, OutBalance ${ }_{k}$ ) is 0. Vice versa, when $\max I D_{k}-\min I D_{k}$ is maximal, i.e., equal to $\mid$ Roles $_{k}-1 \mid$, then the InBalance $_{k}$ is also equal 1 , as well as for OutBalance . $_{\text {. }}$.

Table 3 shows the measure values for the property Efficiency, after having endowed the evaluation framework with the formulae 3 and 4 . The same process has been computed for the properties of Robustness and Flexibility, but for space reasons we do not show the related tables. Subsequently, each configuration has been evaluated against the ideal values of the properties, adopting the criteria described by Definition 3 that results in $R\left(o c_{i}\right), F\left(o c_{i}\right)$ and $E\left(o c_{i}\right)$, as showed in Table 4. At this point, the evaluation criteria takes into account the specific situation to cope with, namely, we have ranked the configurations within each scenario by using Formula 2. To exploit this latter formula over the set of configurations, we have assigned the same importance to each property (i.e., $\rho=\varphi=\eta=\frac{1}{3}$ ), this results in the values within the column labelled $\operatorname{rank}\left(o c_{i}\right)$ of Table 4.

Results for scenarios 1 and 2 are showed in Table 4, but for space reasons we only discuss results of Scenario 2 .

Within Scenario 2, the best configuration results to be $o c_{1}$ that differs from the second best $\left(o c_{4}\right)$ for a symmetric link in the coordination structure between Medics $(M)$ and Service Providers $(S P)$, as illustrated by Figure 3. Notice that, $o c_{1}$ and $o c_{4}$ are two Pareto optimal solutions as $o c_{1}$ performs better over Robustness $\left(R\left(o c_{i}\right)\right)$ and Efficiency $\left(E\left(o c_{i}\right)\right)$, while $O c_{4}$ gives a bigger contribution of satisfaction to Flexibility $\left(F\left(o c_{i}\right)\right)$. Let us assume that within Scenario 2, Flexibility plays a key role to effectively deal with problems. This leads the designer to impose a sensible differentiation over the properties relevance by properly tuning the weights ( $\rho, \varphi$ and $\eta$ ) according to the Formula 2, e.g., $\rho=\eta=\frac{1}{12}$ and $\varphi=\frac{5}{6}$. This latter choice brings $o c_{4}$ in the top of the list $\left(\operatorname{rank}\left(o c_{4}\right)=0,6556\right)$ and $o c_{1}$ in the second best position $\left(\operatorname{rank}\left(o c_{1}\right)=0,6475\right)$.

Notice that, despite the proposed evaluation framework allows to discriminate among several configurations, it does not exactly indicate what graph's links (dependencies) and 
nodes (roles) should be added and/or removed in order to make a configuration better of another one.

\section{Related Work}

The multi-disciplinarity of this paper makes it difficult to provide a comprehensive overview of related work. In this section we briefly recall related work in the area of software engineering.

The work proposed in [2] shares common motivations with ours. Here, the authors propose a design approach to support the development of software systems to dynamically reconfigure and adapt according to contex fluctuations during run time. The authors illustrate their ideas by using some results from a simulation of a grid-enabled wireless sensor network for flood management, which has been deployed in a prototype form to study the flood plain of the River Ribble in North Yorkshire, England.

In [6] the authors use the human organization metaphor to suggest a set of generic organizational structures (e.g., structure-in-5, joint venture, hierarchical contracting) for MASs design on the basis of their degree of satisfaction towards specific software quality attributes such as predictability, security, coordinability and adaptability. On the contrary, our approach is more rigorous because we characterize the organizational properties (robustness, flexibility and efficiency) in terms of structural measures.

In [8] the authors illustrate an interesting solution-also based on the Pareto optimality criterion-for dealing with issues in the area of electrical cable harnessing design. In that work, the main activity consists in designing cable assembly for missiles, airplains and other complex artifacts, which satisfy requirements such as electrical connectivity, routing cables through a three dimensional space with bending and clamping constraints, selecting connectors and optimizing for weight and cost. In our approach, Formula 2 extends the criterion based on the Pareto optimality alone.

In [1] the authors adopt Pareto optimality in order to discriminate among several solutions which fit some constraints (e.g., price, miles, date) with different degree of satisfaction in the domain of online cars selling market.

\section{Conclusions and Future Work}

This paper presents an evaluation framework founded on graph theory and discusses the experimental results given by the framework implementation. The presented approach shows novel ideas on how to characterize organizational configurations merely by means of structural properties they enjoy (formulas of Table 1). It provides a solid and computable theory to measure the degree of adherence of configurations to important organizational properties such as robustness, flexibility and efficiency (Formula 1). Moreover, the approach allows for tuning the relevance of each single property with respect to the scenario at hands (Formula 2 ). The framework has then been evaluated within a crisis management scenario that forces the organization to cope with several possible situations. To this effect, the proposed evaluation criteria support the definition of Pareto optimal configurations within a given situation (Definition 6).

Future research will focus on running several experiments to better tune the right set of parameters that characterize each property.

\section{Acknowledgments}

This work has been performed in the framework of the FP7 project ALIVE IST-215890, which is funded by the European Community. The authors would like to acknowledge the contributions of their colleagues from ALIVE Consortium (http://www.ist-alive.eu).

Davide Grossi acknowledges support from NWO under the VENI grant nr. 639.021.816.

\section{References}

[1] M. Al-Muhammed and D. W. Embley. Resolving underconstrained and overconstrained systems of conjunctive constraints for service requests. In Advanced Information Systems Engineering, 18th International Conference - CAiSE'06, pages 223-238, 2006.

[2] N. Bencomo, P. Grace, C. Flores, D. Hughes, and G. Blair. Genie: Supporting the model driven development of reflective, component-based adaptive systems. In Proc. of 30th International Conference on Software Engineering (ICSE-08), May 10-18 2008.

[3] V. Dignum. A Model for Organizational Interaction: based on Agents, founded in Logic. PhD thesis, Universiteit Utrecht, 2004.

[4] D. Grossi, F. Dignum, M. Dastani, and L. Royakkers. Foundations of organizational structures in multiagent systems. In AAMAS '05: Proceedings of the fourth international joint conference on Autonomous agents and multiagent systems, pages 690-697, New York, NY, USA, 2005. ACM.

[5] D. Grossi, F. Dignum, V. Dignum, M. Dastani, and L. Royakkers. Structural aspects of the evaluation of agent organizations. InCOIN@ECAI 2006, 2006.

[6] M. Kolp, P. Giorgini, and J. Mylopoulos. Multi-Agent Architectures as Organizational Structures. Autonomous Agents and Multi-Agent Systems (JAAMAS), pages 3-25, 2006.

[7] L. Penserini, A. Perini, A. Susi, and J. Mylopoulos. High Variability Design for Software Agents: Extending Tropos. ACM Transactions on Autonomous and Adaptive Systems (TAAS), 2(4), 2007.

[8] C. J. Petrie, T. A. Webster, and M. R. Cutkosky. Using pareto optimality to coordinate distributed agents. Arti Intelligence for Engineering Design, Analysis and Manufacturing (AIEDAM), 9:269-281, 1995. 